\title{
Infinite dimensional Laplacian and spherical harmonics
}

\author{
By \\ Yasuo Umemura and Norio Kôno
}

\begin{abstract}
Summary
The purpose of the present paper is to discuss the properties of the Gaussian measure and the Laplacian operator in infinite dimensions as limits of finite dimensional analogues. In the limit of $n \rightarrow \infty$, the uniform measure on the $n$-dimensional sphere, the spherical Laplacian operator, and Gegenbauer polynomials (spherical harmonics) tend respectively to an infinite dimensional Gaussian measure, the infinite dimensional Laplacian operator, and Hermite polynomials. We also discuss the addition formula and integral representation formula of Hermite polynomials in this limit procedure.
\end{abstract}

\section{Introduction}

In the previous paper [6], one of the authors discussed the infinite dimensional Gaussian measure on the dual space of a nuclear space, for instance, on the space $\left(S^{\prime}\right)$, regarding it as a limit measure of finite dimensional Gaussian measures. It can be also regarded as a limit measure of uniform measures on spheres, as was shown by HidaNomoto [3] : their measure is defined on the projective limit space $\Omega$ of the $n$-dimensional sphere $\Omega_{n}$, but it is equivalent to our measure on $\left(S^{\prime}\right)$, since there exists a measure-preserving one-to-one mapping from $\Omega$ into $\left(\mathcal{S}^{\prime}\right)$.

These two different interpretations reflect the fact that the infinite dimensional Gaussian measure is ergodic with respect to translations like the finite dimensional ones, and also ergodic with respect to

Received October 20, 1965. 
rotations like the uniform measures on spheres.

Next we shall discuss the Laplacian operator. The Laplacian operator $\bar{\Delta}_{n}$ on $\Omega_{n}$ generates all rotationally invariant, symmetric operators on $L^{2}\left(\Omega_{n}, d \omega_{n}\right)$. On the other hand, the infinite dimensional Laplacian operator $\Delta_{c}$ defined in [7] also generates all rotationally invariant operators on $L^{2}\left(\mathcal{S}^{\prime}, \mu_{c}\right)$. (c.f. $\$ 1$ ). Hence, a question arises: does $\bar{\Delta}_{n}$ tend to $\Delta_{c}$ in some sense? In this paper we shall answer it, namely we shall show that $\bar{\Delta}_{n} / n$ tends to $\Delta_{c}$ as $n \rightarrow \infty$.

P. Lévy defined the infinite dimensional Laplacian operator in [5] as the limit operator of $\bar{\Delta}_{n} / n$. Therefore Lévy's definition turns out to be just the same as ours given in [7].

This fact implies the convergency of the eigen values and the eigen functions of $\bar{\Delta}_{n}$ to those of $\Delta_{c}$. Therefore Gegenbauer polynomials tend to Hermite polynomials. This has been known as a formula concerning special functions, for instance see [1]. However, we get here a new interpretation of this formula: finite dimensional spherical harmonics tend to infinite dimensional ones.

Similar new interpretations are possible also for the addition formula and the integral representation formula concerning Hermite polynomials. Especially, we shall show that the density function of the integral representation of the $n$-dimensional zonal spherical function converges to that of the integral representation of Hermite polynomial in the sense of $L^{2}\left(S^{\prime}, \mu_{c}\right)$. The latter representation is equivalent with the Gauss transform.

Our standpoint will make clear some aspects of Hermite polynomials as infinite dimensional eigen functions.

We should also remark that the addition formula played an important role in S. Kakutani's discussions on Brownian motions [4].

In this paper, after preliminary discussions in $\$ 1$, we shall show rather intuitively in $\S 2$ and more exactly in $\S 3$ that the projective limi tof the uniform measures on spheres is just the infinite dimensional Gaussian measure. In $\S 4$, we shall establish the relation between finite 
dimensional and infinite dimensional Laplacian operators, and then investigate how this relation reflects on their eigen functions; for zonal ones in $\$ 5$ and more generally in $\S 6$. In this point of view, we interprete the integral representation formula in $\$ 7$ and the addition formula in $\S 8$.

We would like to thank Prof. H. Yosizawa of Kyoto University and Prof. N. Ikeda of Osaka University for their suggestions of various useful ideas.

\$1. Preliminaries (For details, see [6] and [7]).

Let $L$ be a real nuclear space, and $H$ be its completion by a continuous Hilbertian norm \|\|$_{H}$. Then, we have a relation;

$$
L \subset H \simeq H^{*} \subset L^{*},
$$

where $H^{*}$ and $L^{*}$ are the dual spaces of $H$ and $L$ respectively.

Now, define a function $\chi(\xi)$ on $L$ as follows

$$
\chi(\xi)=\exp \left[-\frac{c^{2}}{2}\|\xi\|_{H}^{2}\right] .
$$

It satisfies the conditions of positive definiteness, continuity in the norm \|\|$_{H}$, and $\chi(0)=1$. So, by the theorem of Bochner-Minlos, $\chi(\xi)$ is the characteristic function of a probability measure $\mu_{c}$ on $L^{*}$ :

$$
\chi(\xi)=\int_{L^{*}} \exp [i\langle T, \xi\rangle] d \mu_{c}(T) .
$$

Here, $\langle T, \xi\rangle$ means the value at $\xi$ of the linear functional $T . \mu_{c}$ is defined on the $\sigma$ - algebra $\mathfrak{B}\left(L^{*}\right)$ which is generated by the family of all Borel cylinder sets of $L^{*}$. We call $\mu_{c}$ a Gaussian measure with variance $c^{2}$.

Definition 1. An unitary operator $u$ on $H$ is called a rotation of $L$, if it satisfies;

i) $u$ maps $L$ onto $L$,

ii) $u$ is homeomorphic on $L$. 
The whole of rotations of $L$ forms a group, which we call the rotation group of $L$ and denote it with $O(L)$. Identifying $u$ with $u^{-1 *}, O(L)$ is regarded as a transformation group on $L^{*}$.

Then, Gaussian measure $\mu_{c}$ is $O$-invariant, i.e. $\mu_{c}$ is invariant under any rotation of $L$. Moreover, it is $O$-ergodic, namely it can not be decomposed into the sum of two measures which are $O$-invariant and singular with each other.

Let $L^{2}\left(L^{*}, \mu_{c}\right)$ be the Hilbert space of all square integrable functions on $L^{*}$ with respect to a Gaussian measure $\mu_{c}$. Denote its inner product and its norm by $(,)_{c}$ and \|\|$_{c}$ respectively.

Let $\xi_{1}, \cdots, \xi_{n}$ be a finite orthonormal system (F.O.N.S.) of $L$ in the norm \|\|$_{H}$. We define the subspace $\mathfrak{U}_{\xi_{1} \cdots \xi_{n}}$ of $L^{2}\left(L^{*}, \mu_{c}\right)$ as follows:

$$
\begin{gathered}
\mathfrak{A}_{\xi_{1} \cdots \xi_{n}}=\left\{F(T) \in L^{2}\left(L^{*}, \mu_{c}\right) \mid \exists f\left(x_{1}, \cdots, x_{n}\right) ;\right. \\
\sqrt{\frac{d \mu_{c}}{d x}}\left(x_{1}, \cdots, x_{n}\right) f\left(x_{1}, \cdots, x_{n}\right) \in(S)_{R^{n}}, \\
\left.F(T)=f\left(\left\langle T, \xi_{1}\right\rangle, \cdots,\left\langle T, \xi_{n}\right\rangle\right)\right\},
\end{gathered}
$$

where $\frac{d \mu_{c}}{d x}=\left(\frac{1}{\sqrt{2 \pi} c}\right)^{n} \exp \left[-\frac{1}{2 c^{2}} \sum_{k=1}^{n} x_{k}^{2}\right]$, and $(S)_{R^{n}}$ is the whole of rapidly decreasing $C^{\infty}$-functions on $R^{n}$ as defined by L. Schwartz.

Put $\mathfrak{A}=\underset{\text { all F.O.N. S. }}{\bigcup} \mathfrak{A}_{\xi_{1} \cdots \xi_{n}}$, then obviously $\mathfrak{A}$ is dense in $L^{2}\left(L^{*}, \mu_{c}\right)$.

Let $\Phi_{\xi_{1} \cdots \xi_{n}}$ be the mapping which maps $f\left(\left\langle T, \xi_{1}\right\rangle, \cdots,\left\langle T, \xi_{n}\right\rangle\right)$ to $f\left(x_{1}, \cdots, x_{n}\right)$.

Definition 2. The infinite dimensional Laplacian operator $\Delta_{c}$ is an operator defined on $\mathfrak{U}$ as follows. If $F(T) \in \mathfrak{U}_{\xi_{1} \cdots \xi_{n}}$, then

$$
\Delta_{c} F(T)=\Phi_{\xi_{1} \cdots \xi_{n}}^{-1} \sum_{i=1}^{n}\left(\frac{\partial^{2}}{\partial x_{i}^{2}}-\frac{x_{i}}{c^{2}} \frac{\partial}{\partial x_{i}}\right) \Phi_{\xi_{1} \cdots \xi_{n}} F(T) .
$$

Since $\sum_{i=1}^{n}\left(\frac{\partial^{2}}{\partial x_{i}^{2}}-\frac{x_{i}}{c^{2}} \frac{\partial}{\partial x_{i}}\right)$ is rotationally invariant, the definition does not depend on the representation of $F(T)$.

$\Delta_{c}$ can be written symbolically as; 


$$
\Delta_{c}=\lim _{n \rightarrow \infty}\left[\Delta_{n}-\frac{r_{n}^{2}}{4 c^{4}}+\frac{n I}{2 c^{2}}\right]
$$

where $A_{n}$ is the usual $n$-dimensional Laplacian operator on $L^{2}\left(R^{n}, d^{n} x\right)$, $r_{n}^{2}=\sum_{k=1}^{n} x_{k}^{2}$ and $I$ is the identity operator.

For an example of a nuclear space, we have the space $(\mathcal{S})$, the whole of rapidly decreasing $C^{\infty}$-functions on the real line. $(\mathcal{S})$ is nuclear because its topology is defined by countable Hilbertian norms;

$$
\|\varphi\|_{p q}=\int_{-\infty}^{\infty}\left(1+x^{2}\right)^{p} \sum_{k=0}^{q}\left|\varphi^{(k)}(x)\right|^{2} d x .
$$

In the above discussions, considering the case that $L=(\mathcal{S})$ and $H=L^{2}\left(R^{1}\right)$, we see that we can define a Gaussian weasure $\mu_{c}$ on $\left(\mathcal{S}^{\prime}\right)$, and the infinite dimensional Laplacian operator $\Delta_{c}$ on $L^{2}\left(S^{\prime}, \mu_{c}\right)$.

\section{\$2. A limit property of uniform measures on spheres}

Let $P_{n}$ be the uniform probability measure on an $n$-dimensional sphere $\Omega_{n}$ of radius $\sqrt{n+1} c$ and center at the origin.

$$
\Omega_{n}: x_{1}^{2}+x_{2}^{2}+\cdots+x_{n+1}^{2}=(n+1) c^{2} .
$$

For this $P_{n}$, consider the joint distribution $P_{n, m}(n \geqq m)$ of $\left(x_{1}, x_{2}\right.$, $\left.\cdots, x_{m}\right)$, namely for $E \subset R^{m}$,

$$
P_{n, m}(E)=P_{n}\left(\left\{\left(x_{1}, \cdots, x_{m}, \cdots, x_{n+1}\right) \mid\left(x_{1}, \cdots, x_{m}\right) \in E\right\}\right) .
$$

\section{Proposition 1 .}

$$
\lim _{n \rightarrow \infty} P_{n, m}(E)=\mu_{c}, m(E)
$$

where $\mu_{c}, n$ is the m-dimensional Gaussian measure with variance $c^{2}$. The convergence is uniform for all Borel subsets $E$ of $R^{m}$.

Proof Decompose $R^{n+1}$ into the sum $R_{1}^{m}+R_{2}^{n-m+1}$, where

and

$$
R_{1}^{m}=\left\{\left(x_{1}, x_{2}, \cdots, x_{m}, o, \cdots, o\right)\right\},
$$

$$
R_{2}^{n-m+1}=\left\{\left(o, \cdots, o, x_{m+1}, x_{n+2}, \cdots, x_{n+1}\right)\right\} \text {. }
$$

Let $x=\left(x_{1}, x_{2}, \cdots, x_{n+1}\right)$ be a point on $\Omega_{n}$, then the distance be- 
tween $x$ and $R_{1}^{m}$ is $\sqrt{(n+1) c^{2}-\left(x_{1}^{2}+\cdots+x_{m}^{2}\right)}$, while the angle $\theta$ between the normal of $\Omega_{n}$ at $x$ and the space $R_{2}^{n-m+1}$ is given by

$$
\frac{1}{\cos \theta}=\frac{\sqrt{n+1} c}{\sqrt{(n+1) c^{2}-\left(x_{1}^{2}+\cdots+x_{m}^{2}\right)}} .
$$

Hence, clearly $P_{n, m}$ is given by

$$
d P_{n, m}=\left\{\begin{array}{cc}
\frac{\sqrt{n+1} c}{\sqrt{(n+1) c^{2}-\left(x_{1}^{2}+\cdots+x_{m}^{2}\right)}} k_{n, m}\left\{(n+1) c^{2}-\left(x_{1}^{2}+\cdots+x_{m}^{2}\right)\right\}^{\frac{n-m}{2}} \\
\times d x_{1} d x_{2} \cdots d x_{m} & \text { for } x_{1}^{2}+\cdots+x_{m}^{2} \leqq(n+1) c^{2} \\
0 & \text { for } x_{1}^{2}+\cdots+x_{m}^{2}>(n+1) c^{2},
\end{array}\right.
$$

where $k_{n, m}$ is the normalization constant. Putting $k_{n, m}\left((n+1) c^{2}\right)^{\frac{n-m}{2}}$ as $k_{n}^{\prime}, m$, we have

$$
d P_{n, m}=k_{n, m}^{\prime}\left(1-\frac{x_{1}^{2}+\cdots+x_{m}^{2}}{(n+1) c^{2}}\right)_{+}^{\frac{n-m-1}{2}} d x_{1} \cdots d x_{m}
$$

where $r_{+}$means $\operatorname{Max}(r, 0)$. From this, we see that

$$
\frac{d P_{n, m}}{k_{n, m}^{\prime} d x_{1} \cdots d x_{m}} \underset{(n \rightarrow \infty)}{\longrightarrow} \exp \left[-\frac{x_{1}^{2}+\cdots+x_{m}^{2}}{2 c^{2}}\right] \text {. }
$$

It is easily seen that the convergence is uniform with respect to $\left(x_{1}, \cdots, x_{m}\right)$. This proves the proposition 1 .

\section{§3. The projective limit space $\Omega$}

In order to make the situation in Proposition 1 clearer, we shall construct the projective limit space $\Omega$ of $\Omega_{n}$, and show that the limit measure $P$ of $P_{n}$ is isomorphic with an infinite dimensional Gaussian measure $\mu_{c}$ defined in $\S 1$.

First, for any $m<n$, we shall define a projection $f_{n, m}$ from $\Omega_{n}$ onto $\Omega_{m}$ as follows;

$$
\begin{gathered}
f_{n, m} \text { maps } \omega^{(n)}=\left(x_{1}^{(n)}, \cdots, x_{n+1}^{(n)}\right) \in \Omega_{n} \\
\text { to } \quad \omega^{(m)}=\left(x_{1}^{(m)}, \cdots, x_{m+1}^{(m)}\right) \in \Omega_{m},
\end{gathered}
$$

where 
Infinite dimensional Laplacian and spherical harmonics 169

$$
x_{i}^{(m)}=\frac{\sqrt{m+1} c x_{i}^{(n)}}{\sqrt{x_{1}^{(n) 2}+\cdots+x_{m+1}^{(n) 2}}} \quad \text { for } 1 \leqq i \leqq m+1
$$

Then, $f_{n, m}$ is a measurable mapping defined almost everywhere on $\Omega_{n}$. (The exceptional set is $\left\{\omega^{(n)} \in \Omega_{n} \mid x_{1}^{(n)}=\cdots=x_{m+1}^{(n)}=0\right\}$ ).

(Remark; If we use the polar coordinates, $f_{n, m}$ is defined as a mapping which maps

$$
\left(\theta_{1}, \cdots, \theta_{m}, \cdots, \theta_{n}\right) \text { on } \Omega_{n} \text { to }\left(\theta_{1}, \cdots, \theta_{m}\right) \text { on } \Omega_{m} .
$$

This expression was used in [3]).

The projection $f_{n, m}$ satisfies the following conditions;

i) $f_{l, n}=f_{l, m} \circ f_{m, n}$ for $l<m<n$

ii) $P_{m}(A)=P_{n}\left(f_{m, n}^{-1}(A)\right)$ for $m<n$ and a Borel subset $A$ of $\Omega_{m}$.

Therefore, according to a theorem due to Bochner, we can construct the projective limit probability space $(\Omega, \mathfrak{B}, P)$. It satisfies the following properties:

P1) $\Omega \subset \prod_{n=1}^{\infty} \Omega_{n}$

P2) $f_{m}=f_{m, n} \circ f_{n}$ for $m<n$.

Here, $f_{n}$ is the restriction of $\pi_{n}$ on $\Omega$, where $\pi_{n}$ is the projection from $\prod_{n=1}^{\infty} \Omega_{n}$ onto $\Omega_{n}$.

P3) $\mathfrak{B}$ is generated by $\bigcup_{n=1}^{\infty} f_{n}^{-1}\left(\mathfrak{B}_{n}\right)$, where $\mathfrak{B}_{n}$ is the whole of Borel subsets of $\Omega_{n}$.

P4) $\quad P\left(f_{n}^{-1}(A)\right)=P_{n}(A)$ for $A \in \mathfrak{B}_{n}$.

Since the coordinate $x_{i}^{(n)}(1 \leqq i \leqq n+1)$ is a function on $\Omega_{n}$, it can be regarded as a function on $\Omega$. We denote this function by $X_{i}^{(n)}(\omega)$, then for any $\omega \in \Omega$,

$$
f_{n}(\omega)=\left(X_{1}^{(n)}(\omega), \cdots, X_{n+1}^{(n)}(\omega)\right) \in \Omega_{n} .
$$

$X_{i}^{(n)}(\omega)$ is a measurable fucntion on $\Omega$ because of $\left.\mathbf{P 3}\right)$. 


\section{Lemma 1.}

i) $\int_{\Omega} X_{i}^{(n)}(\omega) d P(\omega)=0, \quad \forall n, 1 \leqq i \leqq n+1$

ii) $\int_{\Omega} X_{i}^{(n)}(\omega) X_{j}^{(m)}(\omega) d P(\omega)$

$$
=\delta_{i j} c^{2} \sqrt{\frac{n+1}{m+1}} \frac{\Gamma\left(\frac{n+1}{2}\right)}{\Gamma\left(\frac{m+1}{2}\right)} \frac{\Gamma\left(\frac{m+2}{2}\right)}{\Gamma\left(\frac{n+2}{2}\right)}
$$

for $m \leqq n$, where $\Gamma$ is Gamma function.

Proof From $\mathbf{P 4}$ ), we have

$$
\int_{\Omega} X_{i}^{(n)}(\omega) d P(\omega)=\int_{\Omega_{n}} x_{i}^{(n)} d P_{n}\left(\omega^{(n)}\right)=0
$$

Similarly, using $\mathbb{P 2}$ ) also, we see that

$$
\begin{aligned}
\int_{\Omega} X_{i}^{(n)} & (\omega) X_{j}^{(m)}(\omega) d P(\omega) \\
& =\int_{\Omega_{n}} x_{i}^{(n)} \frac{\sqrt{m+1} c x_{j}^{(n)}}{\sqrt{x_{1}^{(n) 2}+\cdots+x_{m+1}^{(n) 2}}} d P_{n}\left(\omega^{(n)}\right) \\
& =\delta_{i j} \sqrt{m+1} c \int_{\Omega_{n}} \frac{x_{1}^{(n) 2}}{\sqrt{x_{1}^{(n)^{2}}+\cdots+x_{m+1}^{(n) 2}}} d P_{n}\left(\omega^{(n)}\right) \\
& =\delta_{i j} \frac{c}{\sqrt{m+1}} \int_{\Omega_{n}} \sqrt{\frac{x_{1}^{(n) 2}+\cdots+x_{m+1}^{(n) 2}}{m}} d P_{n}\left(\omega^{(n)}\right)
\end{aligned}
$$

Substituting (5), we get

$$
\begin{aligned}
& =\delta_{i j} c^{2} \sqrt{\frac{n+1}{m+1}} \int_{0}^{1} t^{m+1}\left(1-t^{2}\right)^{\frac{n-m-2}{2}} d t / \int_{0}^{1} t^{m}\left(1-t^{2}\right)^{\frac{n-m-2}{2}} d t \\
& =\delta_{i j} c^{2} \sqrt{\frac{n+1}{m+1}} B\left(\frac{m+2}{2}, \frac{n-m}{2}\right) / B\left(\frac{m+1}{2}, \frac{n-m}{2}\right) \\
& =\delta_{i j} c^{2} \sqrt{\frac{n+1}{m+1}} \frac{\Gamma\left(\frac{n+1}{2}\right)}{\Gamma\left(\frac{m+1}{2}\right)} \frac{\Gamma\left(\frac{m+2}{2}\right)}{\Gamma\left(\frac{n+2}{2}\right)}
\end{aligned}
$$

The relation (5) is expressed as a formula concerning with $P$ as follows. 
For any $n \geqq m$, and for any Borel subset $E$ of $R^{m}$, we have

$$
\begin{aligned}
P(\{\omega \mid & \left.\left.\left(X_{1}^{(n)}(\omega), \cdots, X_{m}^{(n)}(\omega)\right) \in E\right\}\right) \\
\quad=k_{n}^{\prime}, m & \int_{E}\left(1-\frac{x_{1}^{2}+\cdots+x_{m}^{2}}{(n+1) c^{2}}\right)^{\frac{n-m-1}{2}} d x_{1} \cdots d x_{m} .
\end{aligned}
$$

Lemma 2. $\left\{x_{i}^{(n)}(\omega) ; n=i, i+1, i+2, \cdots \cdots\right\}$ forms a Cauchy sequence in $L^{2}(\Omega, P)$.

Proof From the previous lemma, we get

$$
\begin{aligned}
\left\|X_{i}^{(n)}-X_{i}^{(m)}\right\|_{P}^{2} & =2 c^{2}-2 c^{2} \sqrt{\frac{n+1}{m+1}} \frac{\Gamma\left(\frac{n+1}{2}\right)}{\Gamma\left(\frac{m+1}{2}\right)} \frac{\Gamma\left(\frac{m+2}{2}\right)}{\Gamma\left(\frac{n+2}{2}\right)} \\
& \rightarrow 0 \text { as } n, m \rightarrow \infty,
\end{aligned}
$$

because we have asymptotically

$$
\frac{\Gamma\left(t+\frac{1}{2}\right)}{\Gamma(t)} \sim \sqrt{t} .
$$

From lemma 2, $X_{i}^{(n)}$ converges to a function $X_{i}$ in $L^{2}(\Omega, P)$. Then, $X_{i}(\omega)$ is defined for almost all $\omega$.

Evidently,

$$
\int_{\Omega} X_{i}(\omega) d P(\omega)=0 \text { and } \int_{\Omega} X_{i}(\omega) X_{j}(\omega) d P(\omega)=\delta_{i j} c^{2} .
$$

Next, we shall imbed $\Omega$ into the space $L^{*}$, the dual space of a nuclear space $L$. (c. f. $\S 1$ ).

Since $L$ is nuclear, there exist a complete orthonormal system $\left\{\xi_{k}\right\}$ of $L$ in the norm \|\|$_{H}$ and a square summable sequence $\left\{\lambda_{k}\right\}$ such that the norm:

$$
p(\xi)=\sqrt{\sum_{k=1}^{\infty} \frac{\left(\xi, \xi_{k}\right)_{H}^{2}}{\lambda_{k}^{2}}}
$$

is continuous in the topology of $L$.

Then, we see that for P-almost all $\omega \in \Omega$,

$$
T_{\omega}(\xi) \equiv \sum_{k=1}^{\infty}\left(\xi, \xi_{k}\right)_{H T} X_{k}(\omega)
$$


is defined and continuous on L. Because

$$
\sum_{k=1}^{\infty}\left|\left(\xi, \xi_{k}\right)_{H}\right|\left|X_{k}(\omega)\right| \leqq p(\xi) q(\omega),
$$

where $q(\omega) \equiv \sqrt{\sum_{k=1}^{\infty} \lambda_{k}^{2} X_{k}(\omega)^{2}}$ is finite for $P$-almost all $\omega$.

Consider the mapping $\Psi: \omega \in \Omega \rightarrow T_{\omega} \in L^{*}$. $\Psi$ is one-to-one except on a suitable null-set of $\Omega$, because if $T_{\omega}(\xi)=T_{\omega^{\prime}}(\xi)$ for any $\xi$, we have $X_{k}(\omega)=X_{k}\left(\omega^{\prime}\right)$ for any $k$. This implies $\omega=\omega^{\prime}$, since for $P$-almost all $\omega$, we get from (6)

$$
X_{i}^{(m)}(\omega)=\frac{\sqrt{m+1} c X_{i}(\omega)}{\sqrt{X_{1}(\omega)^{2}+\cdots+X_{m+1}(\omega)^{2}}} .
$$

thus for any $m$ we have $f_{m}(\omega)=f_{m}\left(\omega^{\prime}\right)$.

Next, we shall discuss the measurability. From $\mathbb{P} 3$ ), the probability measure $P$ is defined on the smallest $\sigma$-algebra $\mathfrak{B}$ which makes all $X_{i}^{(n)}(\omega)$ measurable. From (11), this is equivalent to say $\mathfrak{B}$ is the smallest $\sigma$-algebra which makes all $X_{i}(\omega)$ measurable. Therefore, the image $\Psi(\mathfrak{B})$ is the smallest $\sigma$-algebra which makes all $\langle T, \xi\rangle$ measurable regarding $\xi$ as a linear functional on $L^{*}$. In other words, $\Psi(\mathfrak{B})$ is the smallest $\sigma$-algebra which makes all Borel cylinder sets of $L^{*}$ measurable. This means that $\Psi(\mathfrak{B})$ is equal to $\mathfrak{B}\left(L^{*}\right)$ defined in $\S 1$.

Finally, we shall show that the measure $P$ on $\Omega$ is mapped to a Gaussian measure $\mu_{c}$ on $L^{*}$. Because of (8), we get

$$
\begin{aligned}
& P\left[\left\{\omega \mid\left(X_{1}(\omega), \cdots, X_{m}(\omega)\right) \in E\right\}\right] \\
& \quad=\left(\frac{1}{\sqrt{2 \pi} c}\right)^{m} \int_{E} \exp \left[-\frac{x_{1}^{2}+\cdots+x_{m}^{2}}{2 c^{2}}\right] d x_{1} \cdots d x_{m} .
\end{aligned}
$$

So that if $\xi=\sum_{k=1}^{N} \alpha_{k} \xi_{k}$, we have from (10)

$$
\begin{aligned}
& \int_{\Omega} \exp \left[i T_{\omega}(\xi)\right\rfloor d P(\omega) \\
& =\exp \left[-\frac{c^{2}}{2} \sum_{k=1}^{N}\left(\xi, \xi_{k}\right)_{H}^{2}\right]=\exp \left[-\frac{c^{2}}{2}\|\xi\|_{H}^{2}\right] .
\end{aligned}
$$


Since the characteristic function of a measure on $L^{*}$ must be continuous on $L$, we have for any $\xi \in L$,

$$
\int_{\Omega} \exp \left[i T_{\omega}(\xi)\right] d P(\omega)=\exp \left[-\frac{c^{2}}{2}\|\xi\|_{H}^{2}\right] .
$$

This means that $P$ is mapped to $\mu_{c}$, because of one-to-one correspondence between measures and characteristic functions. So far, we have proved:

Proposition 2. The projective limit space $(\Omega, \mathfrak{B}, P)$ is isomorphic with $\left(L^{*}, \mathfrak{B}\left(L^{*}\right), \mu_{c}\right)$.

Namely, there exists a measure-preserving one-to-one mapping from a suitable subset $\widetilde{\Omega}$ of $\Omega$ onto a suitable subset $\widetilde{L}^{*}$ of $L^{*}$ where $P(\widetilde{\Omega})=\mu_{c}\left(\widetilde{L}^{*}\right)=1$.

Remark $L^{*}$ is a vector space, while $\Omega$ is not.

\section{\$4. Laplacian operators}

In this section, we shall prove that the Laplacian operator on $\Omega_{n}$ tends to the infinite dimensional Laplacian operator on $L^{*}$.

On $L^{2}\left(R^{n+1}, d^{n+1} x\right)$, the Laplacian operator is defined by $\Delta_{n+1}=$ $\sum_{i=1}^{n+1} \frac{\partial^{2}}{\partial x_{i}^{2}}$. Using polar coordinates, it is expressed as follows;

$$
\Delta_{n+1}=\frac{\partial^{2}}{\partial r^{2}}+\frac{n}{r} \frac{\partial}{\partial r}+\frac{1}{r^{2}} \bar{\Delta}_{n} .
$$

Here $\overline{\Delta_{n}}$ is the Laplacian operator on the unit sphere;

$$
\begin{aligned}
& \overline{\Delta_{1}}=\frac{\partial^{2}}{\partial \theta_{1}^{2}} \\
& \overline{\Delta_{n}}=\frac{\partial^{2}}{\partial \theta_{n}^{2}}+(n-1) \cot \theta_{n} \frac{\partial}{\partial \theta_{n}}+\frac{1}{\sin ^{2} \theta_{n}} \bar{\Delta}_{n-1} \quad(n \geqq 2)
\end{aligned}
$$

In order to show that the operator $\overline{\Delta_{n}} /(n+1) c^{2}$ tends to $\Delta_{c}$ in some sense, we shall first give a rough discussion, and later formulate it in an exacter way.

We shall operate $\bar{L}_{n} /(n+1) c^{2}$ and $\Delta_{c}$ on a function $f\left(x_{1}, \cdots, x_{m}\right)$, $(m \leqq n)$. We have 


$$
\begin{aligned}
& \Delta_{c} f\left(x_{1}, \cdots, x_{m}\right)=\sum_{i=1}^{m}\left(\frac{\partial^{2}}{\partial x_{i}^{2}}-\frac{x_{i}}{c^{2}} \frac{\partial}{\partial x_{i}}\right) f\left(x_{1}, \cdots, x_{m}\right), \\
& \frac{\bar{\Delta}_{n}}{(n+1) c^{2}} f\left(x_{1}, \cdots, x_{m}\right)=\frac{r^{2}}{(n+1) c^{2}}\left(\sum_{i=1}^{m} \frac{\partial^{2}}{\partial x_{i}^{2}}-\frac{\partial^{2}}{\partial r^{2}}-\frac{n}{r} \frac{\partial}{\partial r}\right) f\left(x_{1}, \cdots, x_{m}\right) .
\end{aligned}
$$

Here, we substitute

$$
\frac{\partial f}{\partial r}=\sum_{i=1}^{m} \frac{x_{i}}{r} \frac{\partial f}{\partial x_{i}}, \frac{\partial^{2} f}{\partial r^{2}}=\sum_{i, j=1}^{m} \frac{x_{i} x_{j}}{r^{2}} \frac{\partial^{2} f}{\partial x_{i} \partial x_{j}},
$$

then we get

$$
\begin{gathered}
\left.\frac{\bar{\Delta}_{n}}{(n+1) c^{2}} f\left(x_{1}, \cdots, x_{m}\right)\right|_{r=\sqrt{n+1} c}=\left[\sum_{i=1}^{m} \frac{\partial^{2}}{\partial x_{i}^{2}}-\frac{1}{(n+1) c^{2}} \sum_{i, j=1}^{m} x_{i} x_{j} \frac{\partial^{2}}{\partial x_{i} \partial x_{j}}\right. \\
\left.-\frac{n}{(n+1) c^{2}} \sum_{i=1}^{n} x_{i} \frac{\partial}{\partial x_{i}}\right] f\left(x_{1}, \cdots, x_{m}\right),
\end{gathered}
$$

thus in the limit of $n \rightarrow \infty$, we see that the right side converges to $\Delta_{c} f\left(x_{1}, \cdots, x_{m}\right)$.

Now, we shall give an exacter expression, regarding both $\overline{\Delta_{n}}$ and $\Delta_{c}$ as operators on $L^{2}\left(L^{*}, \mu_{c}\right)$.

For a fixed C.O.N.S. $\left\{\xi_{k}\right\}$ of $L$, the projective limit space $\Omega$ is imbedded into $L^{*}$ as shown in $\S 3$. For this $\left\{\xi_{k}\right\}$, we consider the space $\mathfrak{A}_{\xi_{1} \cdots \xi_{n}}$ following the definition in $\S 1$, and denote it by $\mathfrak{A}_{n}$. The union $\mathfrak{A}_{\infty} \equiv \bigcup_{n=1}^{\infty} \mathfrak{U}_{n}$ is a subspace of $\mathfrak{A}$ defined in $\S 1$. $\mathfrak{A}_{\infty}$ is also dense in $L^{2}\left(L^{*}, \mu_{c}\right)$.

On the other hand, consider an $n$-dimensional ball $B_{n}$ of radius $\sqrt{n+1} c$ and center at origin.

$$
B_{n}: x_{1}^{2}+\cdots+x_{n}^{2} \leqq(n+1) c^{2} .
$$

Then, the Hilbert space $L^{2}\left(B_{n}, P_{n, n}\right)$, where the measure $P_{n, n}$ is defined by (5), is isomorphic with $L^{2}\left(\Omega_{n}^{+}, 2 P_{n}\right)$, where $2 P_{n}$ is the uniform probability measure on the hemisphere $\Omega_{n}^{+}$.

$$
\Omega_{n}^{+}: x_{1}^{2}+\cdots+x_{n}^{2}+x_{n+1}^{2}=(n+1) c^{2}, x_{n+1}>0 .
$$

$L^{2}\left(\Omega_{n}^{+}, 2 P_{n}\right)$ is a subspace of $L^{2}\left(\Omega_{n}, P_{n}\right)$ which consists of all such functions that satisfy 


$$
f\left(x_{1}, \cdots, x_{n}, x_{n+1}\right)=f\left(x_{1}, \cdots, x_{n},-x_{n+1}\right) .
$$

So, the Laplacian $\bar{\Delta}_{n}$ is defined on $L^{2}\left(\Omega_{n}^{+}, 2 P_{n}\right)$, hence on $L^{2}\left(B_{n}\right.$, $\left.P_{n, n}\right)$ also.

Define the mapping $Q_{n}$ from $L^{2}\left(R^{n}, \mu_{c, n}\right)$ into $L^{2}\left(B_{n}, P_{n, n}\right)$ as follows;

$Q_{n}$ maps any polynomial on $R^{n}$ to its restriction on $B_{n}$.

Since a polynomial on $B_{n}$ is uniquely extended on $R^{n}, Q_{n}$ is oneto-one on the set $\Re_{n}$ of all polynomials of $n$-variables. Remark that $\Re_{n}$ is dense in $L^{2}\left(R^{n}, \mu_{c}, n\right)$ and $Q_{n} \Re_{n}$ is dense in $L^{2}\left(B_{n}, P_{n}, n\right)$.

Now, define an operator $\overline{\bar{\Delta}}_{n}$ on $\Re_{n}$ as follows;

$$
\overline{\overline{\Delta_{n}}}=Q_{n}^{-1} \circ \overline{\Delta_{n}} \circ Q_{n} .
$$

We shall express $\overline{\bar{\Delta}}_{n}$ as a differential operator on $R^{n}$.

On the hemisphere $\Omega_{n}^{+}$:

$$
x_{1}^{2}+\cdots+x_{n}^{2}+x_{n+1}^{2}=(n+1) c^{2}, x_{n+1}>0,
$$

$x_{1}, \cdots, x_{n}$ can be regarded as coordinates. Namely, $x_{1}, \cdots, x_{n}$ are independent variables on $\Omega_{n}^{+}$, and $x_{n+1}=\sqrt{(n+1) c^{2}-x_{1}^{2}-\cdots-x_{n}^{2}}$ is a function of them. Using such coordinates, we have.

$$
\overline{\Delta_{n}}=(n+1) c^{2} \sum_{i=1}^{n} \frac{\partial^{2}}{\partial x_{i}^{2}}-\sum_{i, j=1}^{n} x_{i} x_{j} \frac{\partial^{2}}{\partial x_{i} \partial x_{j}}-n \sum_{i=1}^{n} x_{i} \frac{\partial}{\partial x_{i}} .
$$

This expression is obtained in the following way: In the operator $\Delta_{n+1}$ we change variables from $\left(x_{1}, \cdots, x_{n}, x_{n+1}\right)$ to $\left(\alpha_{1}, \cdots, \alpha_{n}, r\right)$, where $r=\sqrt{x_{1}^{2}+\cdots+x_{n+1}^{2}}$ and $\alpha_{\imath}=x_{i} / r$, then subtract $\frac{\partial^{2}}{\partial r^{2}}+\frac{n}{r} \frac{\partial}{\partial r}$ from it, and finally put $r=\sqrt{n+1} c$.

So, for $f \in \mathfrak{P}_{n}$ we have

$$
\begin{aligned}
\overline{\bar{\Delta}_{n}} f\left(x_{1}, \cdots, x_{n}\right)=(n+1) c^{2} \sum_{i=1}^{n} \frac{\partial^{2} f}{\partial x_{i}^{2}} \\
-\sum_{i, j=1}^{n} x_{i} x_{j} \frac{\partial^{2} f}{\partial x_{i} \partial x_{j}}-n \sum_{i=1}^{n} x_{i} \frac{\partial f}{\partial x_{i}}
\end{aligned}
$$

Thus $\overline{\bar{\Delta}}_{n}$ is continuous in the topology of $(\mathcal{S})_{R^{n}}$. Namely, for a sequence 
$\left\{f_{k}\right\} \subset \mathfrak{\Re}_{n}$, if $f_{k}\left(x_{1}, \cdots, x_{n}\right) \sqrt{\frac{d \mu_{c}}{d x}\left(x_{1}, \cdots, x_{n}\right)}$ tends to 0 in the topology of $(S)_{R^{n}}$, then $\left(\overline{\bar{\Delta}_{n}} f_{k}\right) \sqrt{\frac{d \mu_{c}}{d x}}$ tends also to 0 in $(S)_{R^{n}}$. Therefore, $\overline{\overline{\Delta_{n}}}$ can be extended continuously on $\mathfrak{A}_{n}$.

Consider the mapping $\Phi_{\xi_{1} \ldots \xi_{n}}$ from $\mathfrak{A}_{n}$ into $L^{2}\left(R^{n}, \mu_{c}, n\right)$ following the definition in $\S 1$, and denote it by $\Phi_{n}$. Furthermore, put $\widetilde{\Delta}_{n}=\Phi_{n}^{-1}$ 。 $\overline{\overline{\Delta_{n}}} \circ \Phi_{n} . \quad \widetilde{\Delta}_{n}$ is an operator on $\mathfrak{U}_{n}$. Namely, for $F(T)=f\left(\left\langle T, \xi_{1}\right\rangle, \cdots\right.$, $\left.\left\langle T, \xi_{n}\right\rangle\right) \in \mathfrak{N}_{n}$,

$$
\begin{aligned}
\widetilde{\Delta_{n}} F(T) & =\left[(n+1) c^{2} \sum_{i=1}^{n} \frac{\partial^{2}}{\partial x_{i}^{2}}-\sum_{i, j=1}^{n} x_{i} x_{j} \frac{\partial^{2}}{\partial x_{i} \partial x_{j}}\right. \\
- & \left.n \sum_{i=1}^{n} x_{i} \frac{\partial}{\partial x_{i}}\right]\left.f\left(x_{1}, \cdots, x_{n}\right)\right|_{x_{i}=\left\langle T, \xi_{i}\right\rangle},
\end{aligned}
$$

where $\left.\right|_{x_{i}=\left\langle T, \xi_{i}\right\rangle}$ means that after the calculation of the right hand side we replace $x_{i}$ by $\left\langle T, \xi_{i}\right\rangle$.

Since $\mathfrak{A}_{n} \supset \mathfrak{A}_{m}$ for $n>m, \widetilde{\Delta}_{n}$ is defined also on $\mathfrak{A}_{m}$. So that on the space $\mathfrak{N}_{\infty} \equiv \bigcup_{m=1}^{\infty} \mathfrak{A}_{m}$, we can consider symbolically the limit of $\widetilde{\Delta}_{n} /(n+1) c^{2}$.

Now, we want to prove;

\section{Proposition 3.}

$$
\lim _{n \rightarrow \infty} \frac{\widetilde{\Delta}_{n}}{(n+1) c^{2}}=\Delta_{c} \text { strongly on } \mathfrak{A}_{\infty}
$$

Namely, for any $F \in \mathfrak{A}_{\infty}$,

$$
\lim _{n \rightarrow \infty} \frac{\widetilde{\Delta}_{n}}{(n+1) c^{2}} F=\Delta_{c} F \text { in } L^{2}\left(L^{*}, \mu_{c}\right) .
$$

Proof Since $\mathfrak{A}_{m}$ can be regarded as a subspace of $L^{2}\left(R^{m}, \mu_{c}, m\right)$, it is sufficient to show that

$$
\lim _{n \rightarrow \infty} \frac{\overline{\overline{\Delta_{n}}}}{(n+1) c^{2}} f\left(x_{1}, \cdots, x_{m}\right)=\sum_{i=1}^{m}\left(\frac{\partial^{2}}{\partial x_{i}^{2}}-\frac{x_{i}}{c^{2}} \frac{\partial}{\partial x_{i}}\right) f\left(x_{1}, \cdots, x_{m}\right)
$$

in $L^{2}\left(R^{m}, \mu_{c}, m\right)$.

If the function $f$ does not depend on $x_{m+1} \cdots, x_{n}$, the sum in the 
right hand side of (18) is sufficient to take from 1 to $m$. Therefore, dividing the both hand sides of $(18)$ by $(n+1) c^{2}$, and taking the limit of $n \rightarrow \infty$, the wanted relation (21) is easily obtained.

Moreover, it is clear that in $(20)^{\prime}$, we can take the limit not only in the norm of $L^{2}\left(L^{*}, \mu_{c}\right)$, but also in pointwise way as functions on $L^{*}$. Indeed $(20)^{\prime}$ holds in any meaning of the limit, provided that it makes the space $\mathfrak{A}_{\infty}$ a topological vector space.

\section{§. Zonal spherical functions}

From the result of $\$ 4$, we can expect that spherical functions on $\Omega_{n}$ tend to Hermite polynomials which are eigen functions of $\Delta_{c}$. In this section, we shall assure this for zonal spherical functions.

On $\Omega_{n}$, eigen functions of $\overline{\Delta_{n}}$ are called spherical functions. Especially, a spherical function dependent only on $x_{1}$ is called zonal. It is given by Gegenbauer polynomial as explained below.

From $(18), \overline{\overline{\Delta_{n}}}$ is written on $\Re_{1}$ as follows;

$$
\overline{\overline{\Delta_{n}}} \mid \mathfrak{F}_{1}=\left\{(n+1) c^{2}-x_{1}^{2}\right\} \frac{d^{2}}{d x_{1}^{2}}-n x_{1} \frac{d}{d x_{1}}
$$

Its eigen values are $-l(l+n-1), l=0,1,2, \cdots$, and the corresponding eigen functions are

$$
f_{l,}\left(x_{1}\right) \equiv C_{l}^{\frac{n-1}{2}}\left(\frac{x_{1}}{\sqrt{n+1} c}\right)
$$

Here, $C_{l}^{p}(x)$ is Gegenbauer polynomial. It is defined as the solution of

$$
\left[\left(1-x^{2}\right) \frac{d^{2}}{d x^{2}}-(2 p+1) x \frac{d}{d x}+l(l+2 p)\right] C_{l}^{p}(x)=0
$$

which satisfies $C_{l}^{p}(1)=(2 p+l-1) ! / l !(2 p-1) !$.

On the other hand, $\Delta_{c}$ is written on $\mathfrak{P}_{1}$ as follows;

$$
\Delta_{c} \mid \mathfrak{P}_{1}=\frac{d^{2}}{d x_{1}^{2}}-\frac{x_{1}}{c^{2}} \frac{d}{d x_{1}} .
$$


Its eigen values are $-l / c^{2}, l=0,1,2, \cdots$, and the corresponding eigen functions are

$$
f_{l}\left(x_{1}\right)=H_{l}\left(\frac{x_{1}}{\sqrt{2} c}\right)
$$

Here, $H_{l}(x)$ is Hermite polynomial. It is defined as the solution of

$$
\left[\frac{d^{2}}{d x^{2}}-2 x \frac{d}{d x}+2 l\right] H_{l}(x)=0
$$

whose coefficient of $x^{l}$ (=the highest degree) is $2^{l}$.

Therefore, on $\Re_{1}$ the result in $\$ 4$ means that

$$
\left(1-\frac{x_{1}^{2}}{(n+1) c^{2}}\right) \frac{d^{2}}{d x_{1}^{2}}-\frac{n x_{1}}{(n+1) c^{2}} \frac{d}{d x_{1}} \rightarrow \frac{d^{2}}{d x_{1}^{2}}-\frac{x_{1}}{c^{2}} \frac{d}{d x_{1}}
$$

strongly on $\mathfrak{P}_{1}$.

Corresponding to this, the eigen values $\lambda_{l, n} \equiv-\frac{l(l+n-1)}{(n+1) c^{2}}$ tend to $\lambda_{l} \equiv-\frac{l}{c^{2}}$, and the eigen functions $f_{l},{ }_{n}\left(x_{1}\right)$ also converge to $f_{l}\left(x_{1}\right)$ except the normalization constants.

The latter result is easily verified by comparing the coefficients in the polynomial $f_{l}\left(x_{1}\right)$ whih those in $f_{l, n}\left(x_{1}\right)$. But, we can regard it as a direct result of the fact $\frac{\widetilde{\Delta}_{n}}{(n+1) c^{2}} \rightarrow \Delta_{c}$.

More exactly speaking, let $\mathfrak{P}_{1, \text { }}$ be the set of all polynomials of one variable with degrees at most $l$. $\mathfrak{\Re}_{1, l}$ is an $(l+1)$-dimensional vector space. Both the operators $\overline{\bar{\Delta}}_{n}$ and $\Delta_{c}$ keep $\Re_{1, l}$ invariant, so that they are represented as matrices on $\mathfrak{F}_{1, l}$.

Lemma 3. If a $k \times k$-matrix $A$ has $k$ different eigen values, and if $k \times k$-matrices $A_{n}$ tend to $A$, then for sufficiently large $n$. $A_{n}$ also has $k$ different eigen values. Moreover, eigen values and eigen vectors of $A_{n}$ tend to those of $A$ respectively.

Proof is omitted. 
Since $\Delta_{c}$ has $l+1$ different eigen values on $\mathfrak{P}_{1, l}$, we have the following proposition. The ratio of the normalization constants is determined by comparing the coefficients of $x^{l}$.

\section{Proposition 4.}

$$
\lim _{n \rightarrow \infty} \frac{l ! 2^{l / 2}}{n^{l / 2}} C_{l}^{n / 2}\left(\frac{x}{\sqrt{n}}\right)=H_{l}\left(\frac{x}{\sqrt{2}}\right) .
$$

Our new interpretation of this formula is that n-dimensional zonal spherical functions tend to infinite dimensional ones.

\section{§6. Spherical functions of many variables.}

In this section, generalizing the result in $\$ 5$, we shall establish the relation between finite dimensional and infinite dimensional spherical functions which are not necessarily zonal.

As we have discussed in [7], the infinite dimensional Laplacian operator $\Delta_{c}$ on $L^{2}\left(L^{*}, \mu_{c}\right)$ has eigen values $-l / c^{2}, l=0,1,2, \cdots$. For the fixed C.O.N.S. $\left\{\xi_{k}\right\}$ of $L$, the eigen space $\mathfrak{S}_{l}$ is spanned by

$$
F_{l_{1} l_{2} \cdots l_{k} \cdots}(T) \equiv \prod_{k=1}^{\infty} H_{l_{k}}\left(\frac{\left\langle T, \xi_{k}\right\rangle}{\sqrt{2} c}\right),
$$

where $l_{1}+l_{2}+\cdots+l_{k}+\cdots=l$. Consequently, except for $l=0$, each eigen value $-l / c^{2}$ has infinite multiplicity.

In order to resolve this multiplicity, let us consider the partial Laplacians $\Delta_{c}^{(R)}$. Let $R$ be a finite dimensional subspace of $L$, and suppose that some elements of a C.O.N.S. $\left\{\xi_{k}\right\}$ of $L$, say $\xi_{1}, \xi_{2}, \cdots, \xi_{\nu}$, form a C.O.N.S. of $R$.

Then, for $F(T) \in \mathfrak{A}_{\xi_{1} \xi_{2} \cdots \xi_{n}}(n>\nu)$, we put

$$
\Delta_{c}^{(R)} F(T)=\Phi_{\xi_{1} \ldots \xi_{n}}^{-1} \sum_{i=\nu+1}^{n}\left(\frac{\partial^{2}}{\partial x_{i}^{2}}-\frac{x_{i}}{c_{2}} \frac{\partial}{\partial x_{i}}\right) \Phi_{\xi_{1} \cdots \xi_{n}} F(T) .
$$

$\Delta_{c}^{(R)}$ is defined consistently by (30), independent from the choice of a C.O.N.S. $\left\{\xi_{k}\right\}$ provided that its first $\nu$ members form a C.O.N.S. of $R$.

Now, we shall consider the fixed C.O.N.S. $\left\{\xi_{k}\right\}$ of $L$. We shall 
denote $\Delta_{c}^{(\nu)}$ instead of $\Delta_{c}^{(R)}$, when the subspace $R$ is spanned by $\xi_{1}, \cdots, \xi_{\nu}$. Then, it is evident that

$$
\Delta_{c}^{(\nu)} F_{l_{1} l_{2} \cdots l_{k} \cdots}(T)=-\frac{l_{\nu+1}+l_{\nu+2}+\cdots}{c^{2}} F_{l_{1} l_{2} \cdots l_{k} \cdots}(T) .
$$

Therefore, $F_{l_{1} l_{2} \cdots l_{k} \ldots}(T)$ is a simultaneous eigen function of the family of operators $\Delta_{c}^{(\nu)}, \nu=0,1,2, \cdots$. (We put $\Delta_{c}^{(0)} \equiv \Delta_{c}$ ). Furthermore, each simultaneous eigen value is simple.

Namely, for any decreasing sequence $\left\{\lambda_{\nu}\right\}$ of non-negative integers which tend to zero;

$$
\lambda_{0} \geqq \lambda_{1} \geqq \lambda_{2} \geqq \cdots \rightarrow 0, \quad \text { each } \lambda_{\nu} \text { is an integer, }
$$

there exists the unique simultaneous eigen function of $\Delta_{c}^{(v)}$ whose eigen value is $-\lambda_{\nu} / c^{2}$. It is given by $F_{l_{1} l_{2} \cdots l_{k} \cdots}(T)$ where $\lambda_{\nu}=l_{\nu+1}+l_{\nu+2}+\cdots$, or $l_{k}=\lambda_{k-1}-\lambda_{k}$.

Next, we shall consider the partial Laplacians on spheres. Usually, they are expressed in terms of polar coordinates, but here we shall adopt the coordinates used in $\S 4$.

Namely, we regard $x_{1}, \cdots, x_{n}$ as independent variables on $\Omega_{n}^{+}$, and $x_{n+1}=\sqrt{(n+1) c^{2}-x_{1}^{2}-\cdots-x_{n}^{2}}$ as a function of them. Then, using this coordinates the partial Laplacians on the hemisphere $\Omega_{n}^{+}$is defined by:

$$
\begin{aligned}
\bar{\Delta}_{n}^{(\nu)}= & \left((n+1) c^{2}-x_{1}^{2}-\cdots-x_{\nu}^{2}\right) \sum_{i=\nu+1}^{n} \frac{\partial^{2}}{\partial x_{i}^{2}} \\
& -\sum_{i, j=\nu+1}^{n} x_{i} x_{j} \frac{\partial^{2}}{\partial x_{i} \partial x_{j}}-(n-\nu) \sum_{i=\nu+1}^{n} x_{i} \frac{\partial}{\partial x_{i}} .
\end{aligned}
$$

Remark that $\overline{\Delta_{n}^{(0)}}=\overline{\Delta_{n}}$.

It is well known that the simultaneous eigen function of $\bar{\Delta}_{n}^{(v)}$ $(\nu=0,1, \cdots, n-1)$ is given by a product of Gegenbauer polynomials. Regarding $x_{1}, \cdots, x_{n}$ as independent variables on $\Omega_{n}^{+}$, it is written as follows; 


$$
\begin{gathered}
Y_{n, l_{1} l_{2} \cdots l_{n}}\left(x_{1}, \cdots, x_{n}\right) \equiv \prod_{k=1}^{n}\left\{(n+1) c^{2}-x_{1}^{2}-\cdots-x_{k-1}^{2}\right\}^{l_{k} / 2} \\
\times C_{l_{k}}^{\frac{1}{2}(n-k)+\lambda_{k}}\left(\frac{x_{k}}{\sqrt{(n+1) c^{2}-x_{1}^{2}-\cdots-x_{k-1}^{2}}}\right),
\end{gathered}
$$

where $\lambda_{k}=l_{k+1}+\cdots+l_{n}$.

This function satisfies;

$$
\bar{\Delta}_{n}^{(\nu)} Y_{n, l_{1} l_{2} \cdots l_{n}}=-\lambda_{\nu}\left(\lambda_{\nu}+n-\nu-1\right) Y_{n, l_{1} l_{2} \cdots l_{n}} .
$$

The set $\left\{Y_{n, l_{1} l_{2} \cdots l_{n}} ; l_{k}=0,1,2, \cdots\right\}$ forms a C.O.S. of $L^{2}\left(\Omega_{n}^{+}, 2 P_{n}\right)$.

In the same way as in $\S 4$, the operators $\overline{\overline{\Delta_{n}^{(\nu)}}}$ are defined on $L^{2}\left(R^{n}, \mu_{c}, n\right) . \quad \overline{\bar{\Delta}_{n}^{(v)}}$ is also given by the right hand side of (33), so that regarding $Y_{n, l_{1} l_{2} \cdots l_{n}}$ as a polynomial on $R^{n}$, it is a simultaneous eigen function of $\overline{\overline{\Delta_{n}^{(\nu)}}}$. The operators $\widetilde{\Delta_{n}^{(\nu)}}$ are also defined on $L^{2}\left(L^{*}, \mu_{c}\right)$, and their simultaneous eigen function $F_{n, l_{1} l_{2} \cdots l_{n}}(T)$ is obtained by substituting $x_{i}=\left\langle T, \xi_{i}\right\rangle$ in the right hand side of (34).

It is easily seen that on the space $\mathscr{2}_{\infty}$ defined in $\$ 4$, the operator $\widetilde{\Delta_{n}^{(\nu)}} /(n+1) c^{2}$ tends strongly to $\Delta_{c}^{(\nu)}$ as $n \rightarrow \infty$. Corresponding to this fact, the former's eigen value $-\frac{\lambda_{\nu}\left(\lambda_{\nu}+n-\nu-1\right)}{(n+1) c^{2}}$ tends to the latter's eigen value $-\lambda_{v} / c^{2}$.

The space $\mathfrak{P}_{m, l}$, the whole of polynomials of $m$ variables with degrees at most $l$, becomes a vector space. Its dimension is $d_{m, l}=$ $\sum_{k=0}^{l}\left(\begin{array}{c}k+m-1 \\ k\end{array}\right)$.

Regarding $\mathfrak{P}_{m, l}$ as a subspace of $\mathfrak{A}_{m}$, we see that on $\mathfrak{P}_{m}$, the set of the partial Laplacians $\Delta_{c}^{(0)}, \Delta_{c}^{(1)}, \cdots, \Delta_{c}^{(m-1)}$ has $d_{m, l}$-different sets of eigen values $\left\{-\lambda_{\nu} / c^{2} ; 0 \leq \nu \leq m-1\right\}$;

$$
l \geqq \lambda_{0} \geqq \lambda_{1} \geqq \cdots \geqq \lambda_{m-1} \geqq 0 .
$$

So that, generalizing Lemma 3 in $\S 5$ to the case of simultaneous eigen values, we can show that a suitable spherical function tends to $F_{l_{1} l_{2} \cdots l_{k} \ldots}(T)$.

For $n \geqq m>\nu$, the operator $\overline{\overline{\Delta_{n}^{(\nu)}}}$ is defined on $\mathfrak{F}_{m, l}$, and in the limit of $n \rightarrow \infty$ it tends strongly to $\Delta_{c}^{(\nu)}$. Therefore, we get the following proposition. The ratio of the normalization constants is obtained 
by comparing the coefficients of $\prod_{k=1}^{\infty} x_{k}^{l_{k}}$.

\section{Proposition 5.}

$$
\begin{gathered}
\lim _{n \rightarrow \infty}\left[l_{1} ! l_{2} ! \cdots l_{n} ! \frac{2^{l / 2}}{(n c)^{l}} Y_{n, l_{1} l_{2} \cdots l_{n}}\left(x_{1}, \cdots, x_{n}\right)\right] \\
=\prod_{k=1}^{\infty} H_{l_{k}}\left(\frac{x_{k}}{\sqrt{2} c}\right),
\end{gathered}
$$

where $l=l_{1}+l_{2}+\cdots+l_{n}+\cdots<\infty$.

Though this formula is obtained directly from Prop. 4, we have its new interpretation; the simultaneous eigen function of the partial Laplacians on the n-dimensional sphere tends to the infinite dimensional one.

Remark In general, for a sequence of C.O.N.S. $\left\{\xi_{k}^{(n)}\right\}$, even if $\lim _{n \rightarrow \infty}\left\{\xi_{k}^{(n)}\right\}=\xi_{k}$ strongly for any $k$, the limit O.N.S. $\left\{\xi_{k}\right\}$ is not always complete.

But in our case, a finite dimensional subspace $\mathfrak{P}_{m, l}$ is invariant both under $\overline{\overline{\Delta_{n}^{(\nu)}}}$ and $\Delta_{c}^{(\nu)}$, and $\bigcup_{m, l=1}^{\infty} \Re_{m}, l$ is dense in $L^{2}\left(L^{*}, \mu_{c}\right)$. Therefore, from the completeness of the system $\left\{Y_{n, l_{1} l_{2} \cdots l_{n}}\right\}$ in $L^{2}\left(\Omega_{n}^{+}, 2 P_{n}\right)$, we can conclude that the system $\left\{\prod_{k=1}^{\infty} H_{l k}\left(\frac{\left\langle T, \xi_{k}\right\rangle}{\sqrt{2} c}\right) ; l_{1}+l_{2}+\cdots<\infty\right\}$ is complete in $L^{2}\left(L^{*}, \mu_{c}\right)$.

\section{§7. Integral representation formula}

For Gegenbauer polynomial, we have the following formula. (c.f. [2] page 177)

$$
C_{l}^{n / 2}(u)=\frac{\Gamma(n+l)}{2^{n-1} l ![\Gamma(n / 2)]^{2}} \int_{-1}^{1}\left(u-i t \sqrt{1-u^{2}}\right)^{l}\left(1-t^{2}\right)^{\frac{n-2}{2}} d t
$$

We can obtain this formula in a natural way, if we express the right side as an integral on the sphere $\Omega_{n}$.

It is evident that if $a_{0}^{2}=a_{1}^{2}+\cdots+a_{n}^{2}$, the polynomial $f_{l, a_{0} \cdots a_{n}}\left(x_{1}, \cdots\right.$, $\left.x_{n+1}\right) \equiv\left(a_{0} x_{1}-i a_{1} x_{2}-\cdots-i a_{n} x_{n+1}\right)^{l}$ is homogeneous and harmonic on $R^{n+1}$. Thus, regarding it as a function on $\Omega_{n}$, it is a spherical harmonics with degree $l$. Namely, it satisfies 


$$
\overline{\Delta_{n}} f_{l, a_{0} \cdots a_{n}}=-l(l+n-1) f_{l, a_{0} \cdots a_{n}} .
$$

This is also true for any linear combination of $f_{l, a_{0} \cdots a_{n}}$.

Let $\omega^{\prime}=\left(x_{1}^{\prime}, \cdots, x_{n}^{\prime}\right)$ be a point on $\Omega_{n-1}$, then being $x_{1}^{\prime 2}+\cdots+x_{n}^{\prime 2}$ $=n c^{2}$, the polynomial of $x_{1}, \cdots, x_{n}$ defined as

$$
\int_{\Omega_{n-1}}\left(\sqrt{n} c x_{1}-i x_{1}^{\prime} x_{2}-\cdots-i x_{n}^{\prime} x_{n+1}\right)^{l} d m\left(\omega^{\prime}\right)
$$

is a spherical function on $\Omega_{n}$, where $m\left(\omega^{\prime}\right)$ is a measure on $\Omega_{n-1}$.

Especially if we consider the uniform measure $P_{n-1}\left(\omega^{\prime}\right)$ on $\Omega_{n-1}$, the integral (38) depends only on $x_{1}$ because of rotationally invariance of $P_{n-1}$, therefore the obtained polynomial becomes zonal. This must be a constant multiple of $C_{l}^{\frac{n-1}{2}}\left(\frac{x_{1}}{\sqrt{n+1 c}}\right)$.

$$
\begin{aligned}
& C_{l}^{\frac{n-1}{2}}\left(\frac{x_{1}}{\sqrt{n+1} c}\right) \propto \int_{\Omega_{n-1}}\left(\sqrt{n} c x_{1}-i x_{1}^{\prime} x_{2}-\cdots-i x_{n}^{\prime} x_{n+1}\right)^{l} d P_{n-1}\left(\omega^{\prime}\right) \\
& \quad \propto \int_{-\sqrt{n} c}^{\sqrt{n} c}\left(\sqrt{n} c x_{1}-i t \sqrt{(n+1) c^{2}-x_{1}^{2}}\right)^{\prime}\left(1-\frac{t^{2}}{n c^{2}}\right)^{\frac{n-3}{2}} d t
\end{aligned}
$$

Putting $x_{1} / \sqrt{n+1} c=u$ and replacing $n-1$ by $n$, we have

$$
C_{l}^{n / 2}(u) \propto \int_{-1}^{1}\left(u-i t \sqrt{1-u^{2}}\right)^{l}\left(1-t^{2}\right)^{\frac{n-2}{2}} d t .
$$

From this, comparing the value at $u=1$, we get (37).

Thus the integral representation formula (37) takes a more natural form by rewriting it into;

$$
\begin{gathered}
C_{l}^{\frac{n-1}{2}}\left(\frac{x_{1}}{\sqrt{n+1} c}\right)=\frac{\sqrt{\pi} \Gamma(n+l-1)}{n^{l / 2}(n+1)^{l / 2} c^{2 l} 2^{n-2} l ! \Gamma\left(\frac{n}{2}\right) \Gamma\left(\frac{n-1}{2}\right)} \\
\quad \times \int_{\Omega_{0: 0-1}}\left(\sqrt{n} c x_{1}-i x_{1}^{\prime} \sqrt{\left.(n+1) c^{2}-x_{1}^{2}\right)^{l}} d P_{n-1}\left(\omega^{\prime}\right)\right.
\end{gathered}
$$

The right hand side is also written as an integral on $\Omega$ as follows:

$$
\begin{aligned}
& \frac{\sqrt{\pi} \Gamma(n+l-1)}{(n+1)^{l / 2} c^{l} 2^{n-2} l ! \Gamma\left(\frac{n}{2}\right) \Gamma\left(\frac{n-1}{2}\right)} \\
& \times \int_{\Omega}\left(x_{1}-i \frac{X_{1}^{(n+1)}(\omega)}{\sqrt{n} c} \sqrt{(n+1) c^{2}-x_{1}^{2}}\right)^{l} d P(\omega) .
\end{aligned}
$$


Letting $n \rightarrow \infty$ in both sides of $(39)^{\prime}$ we shall obtain the integral representation formula of Hermite polynomial. As we have shown in $\S 5$,

$$
\frac{l ! 2^{l / 2}}{n^{l / 2}} C_{l}^{\frac{n-1}{2}}\left(\frac{x_{1}}{\sqrt{n+1} c}\right) \text { tends to } H_{l}\left(\frac{x_{1}}{\sqrt{2} c}\right)
$$

On the other hand, the integrand of $(39)^{\prime \prime}$ converges to $\left(x_{1}-i X_{1}(\omega)\right)^{l}$ in $L^{2}(\Omega, P)$. This comes from the fact that $\left(X_{1}^{(n)}(\omega)\right)^{k}$ converges to $\left(X_{1}(\omega)\right)^{k}$ in $L^{2}(\Omega, P)$ for any $k$. The latter is proved using a generalization of Lemma 1 in $\S 3$.

Lemma 1'.

$$
\begin{aligned}
& \int_{\Omega}\left(X_{1}^{(n)}(\omega)\right)^{\prime}\left(X_{1}^{(m)}(\omega)\right)^{k} d P(\omega) \\
= & c^{j+k}(n+1)^{j / 2}(m+1)^{k / 2} \frac{\Gamma\left(\frac{j+k+1}{2}\right)}{\Gamma\left(\frac{1}{2}\right)} \frac{\Gamma\left(\frac{n+1}{2}\right)}{\Gamma\left(\frac{j+n+1}{2}\right)} \frac{\Gamma\left(\frac{j+m+1}{2}\right)}{\Gamma\left(\frac{j+k+m+1}{2}\right)}
\end{aligned}
$$

for $m \leqq n$ and $j+k=$ even. In the limit of $n, m \rightarrow \infty$, the right hand side tends to $1 \cdot 3 \cdot 5 \cdots(j+k-1) c^{j+k}$.

Proof of Lemma $\mathbf{1}^{\prime}$ is omitted.

By the isomorphism between $L^{2}(\Omega, P)$ and $L^{2}\left(L^{*}, \mu_{c}\right)$, the function $X_{1}(\omega)$ corresponds to $\left\langle T, \xi_{1}\right\rangle$ therefore from (39)" we have

$$
H_{l}\left(\frac{x_{1}}{\sqrt{2} c}\right)=\frac{2^{l / 2}}{c^{l}} \int_{L^{*}}\left(x_{1}-i\left\langle T, \xi_{1}\right\rangle\right)^{l} d \mu_{c}(T) .
$$

We obtain so called Gauss transform rewriting (40) into the form;

$$
\begin{aligned}
H_{l}(u) & =\frac{2^{l / 2}}{\sqrt{2 \pi} c^{l+1}} \int_{-\infty}^{\infty}(\sqrt{2} c u-i t)^{l} \exp \left(-\frac{t^{2}}{2 c^{2}}\right) d t \\
& =\frac{2^{l}}{\sqrt{\pi}} \int_{-\infty}^{\infty}(u-i t)^{l} \exp \left(-t^{2}\right) d t .
\end{aligned}
$$

So far we have proved;

Proposition 6. The density of the integral representation of the zonal spherical function on n-dimensional sphere tends to that of Hermite Polynomial in the sense of $L^{2}\left(L^{*}, \mu_{c}\right)$. 
Remark Since a Gaussian measure is rotationally invariant, for any $\xi \in L$ such that $\|\xi\|=1$, we have;

$$
(40)^{\prime} \quad H_{l}\left(\frac{x_{1}}{\sqrt{2} c}\right)=\frac{2^{l / 2}}{c^{l}} \int_{L^{*}}\left(x_{1}-i\langle T, \xi\rangle\right)^{l} d \mu_{c}(T) .
$$

In other words

$$
(40)^{\prime \prime} \quad H_{l}\left(\frac{\langle T, \xi\rangle}{\sqrt{2 c}}\right)=\frac{2^{l / 2}}{c^{l}} \int_{L^{*}}\langle T-i S, \xi\rangle^{l} d \mu_{c}(S) .
$$

\section{§. Addition formula}

From (40)", if we put $\xi=\xi_{1} \cos \theta+\xi_{2} \sin \theta$, we get

$$
\begin{aligned}
& H_{l}\left(\frac{\left\langle T, \xi_{1}\right\rangle}{\sqrt{2} c} \cos \theta+\frac{\left\langle T, \xi_{2}\right\rangle}{\sqrt{2} c} \sin \theta\right) \\
& \quad=\frac{2^{l / 2}}{c^{l}} \int_{L^{*}}\left(\cos \theta\left\langle T-i S, \xi_{1}\right\rangle+\sin \theta\left\langle T-i S, \xi_{2}\right\rangle\right)^{l} d \mu_{c}(S) .
\end{aligned}
$$

The integrand is written as

$$
\sum_{k=0}^{l}\left(\begin{array}{l}
l \\
k
\end{array}\right) \cos ^{k} \theta \sin ^{l-k} \theta\left\langle T-i S, \xi_{1}\right\rangle^{k}\left\langle T-i S, \xi_{2}\right\rangle^{l-k}
$$

Moreover the distributions of $\left\langle S, \xi_{1}\right\rangle$ and $\left\langle S, \xi_{2}\right\rangle$ are mutually independent. Thus after the integration we get

$$
H_{l}\left(x_{1} \cos \theta+x_{2} \sin \theta\right)=\sum_{k=0}^{l}\left(\begin{array}{l}
l \\
k
\end{array}\right) \cos ^{k} \theta \sin ^{l-k} \theta H_{k}\left(x_{1}\right) H_{l-k}\left(x_{2}\right) .
$$

This is the addition formula of Hermite polynomial. As we have seen here, it is obtained very easily if we regard Hermite polynomial as an infinite dimensional spherical function.

Putting

$$
H_{l}(u, \sigma)=\frac{\sigma^{l / 2}}{l ! 2^{l / 2}} H_{l}\left(\frac{u}{\sqrt{2 \sigma}}\right),
$$

(41) is written as

$$
H_{l}(u+v, \sigma+\tau)=\sum_{k=0}^{l} H_{k}(u, \sigma) H_{l-k}(v, \tau) .
$$

This is the formula of S. Kakutani [4] which plays an essential role in his paper. 
Similarly, from the relation;

$$
\prod_{k=1}^{m} H_{l_{k}}\left(\frac{\left\langle T, \xi_{k}\right\rangle}{\sqrt{2} c}\right)=\frac{2^{l / 2}}{c^{l}} \int_{L^{*}} \prod_{k=1}^{m}\left\langle T-i S, \xi_{k}\right\rangle^{l_{k}} d \mu_{\mu_{c}}(S),
$$

we shall have an addition formula of a product of Hermite polynomials. Since it is too complicated, we present the case $m=2$ :

$$
\begin{gathered}
H_{l}\left(x_{1} \cos \theta+x_{2} \sin \theta\right) H_{k}\left(-x_{1} \sin \theta+x_{2} \cos \theta\right) \\
=\sum_{j=0}^{l+k} \alpha_{j} H_{j}\left(x_{1}\right) H_{l+k-j}\left(x_{2}\right)
\end{gathered}
$$

where

$$
\alpha_{j}=\sum_{i=\operatorname{Max}(0, j-k)}^{\operatorname{Min}(j, l)}\left(\begin{array}{l}
l \\
i
\end{array}\right)\left(\begin{array}{c}
k \\
j-i
\end{array}\right)(-1)^{j-i} \cos ^{k-j+2 i} \theta \sin ^{j+l-2 i} \theta .
$$

Remark that this is not obtained directly from (41).

For Gegenbauer polynomial, we can not derive the addition formula directly from its integral representation. However, it is seen that the addition formula of Gegenbauer polynomial tends term by term to that of Hermite polynomial. The meaning of this fact is well understood if we regard the addition formula as a transformation of an orthogonal base in $L^{2}\left(L^{*}, \mu_{c}\right)$.

\section{REFERENCES}

[1] Appell et Kampé de Fériat, Fonctions hypergéometriques et hypersphériques. Especially page 335(6). Gauthier-Villars (1926).

[2] Bateman and others, Higher transcendental functions. vol. II Chap X, XI. McGraw-Hill (1953).

[3] T. Hida and H. Nomoto, Gaussian measure on the projective limit space of spheres. Proc. Japan Acad. vol. 40 No. 5 (1964) 301-304.

[4] S. Kakutani, Determination of the spectrum of the flow of Brownian motion. Proc. Nat. Acad. Sci. U.S.A. 36 (1950) 319-323.

[5] P. Lévy, Problemes concrets d'analyse fonctionnelle. Troisiéme partie. Gauthier-Villars (1951).

[6] Y. Umemura, Measures on infinite dimensional vector spaces. Publ. of Research Institute for Mathematical Sciences, Kyoto University. vol. 1 No. 1 (1965) 1-47.

[7] Y. Umemura, On the infinite dimensional Laplacian operator. J. Math. Kyoto Univ. vol. 4 No. 3. (1965) 477-492. 